www.nature.com/pj

\title{
Design of epigenetics control carrier for simultaneous transfection of histone acetyltransferase with histone deacetylase inhibitor to continuous histone acetylation
}

\author{
Polymer Journal (2016) 48, 561-564; doi:10.1038/pj.2015.129; published online 27 January 2016
}

\section{INTRODUCTION}

The word 'epigenetics' includes any process that alters gene activity without changing the DNA sequences. ${ }^{1,2}$ Epigenetic modifications such as DNA methylation and histone modification such as acetylation, methylation and phosphorylation have key roles in controlling chromatin structures. ${ }^{3,4}$ Epigenetics is linked to cell differentiation processes such as pluripotency, lineage commitment and developmental processes. ${ }^{5}$ Furthermore, various acquired diseases such as several cancers, type 2 diabetes and neurological disorders occur as a result of complex epigenetic changes caused by the aging process and environmental factors. ${ }^{6-9}$ Although the mechanism of pathogenesis is becoming clear, treatment options for these diseases are limited.

Several examples of epigenetics-based clinical applications are described below. First, suberoylanilide hydroxamic acid as a histone deacetylase inhibitor and 5-aza-deoxycytidine as a DNA methyltransferase inhibitor, have been approved by the FDA as an anti-cancer agents. ${ }^{10-13}$ Second, epigenetics techniques are highly applicable to therapies involving induced pluripotent stem cells. For example, valproic acid is a histone deacetylase (HDAC) inhibitor that improves reprogramming efficiencies by $>100$-fold. ${ }^{14}$ Furthermore, pluripotent stem cells can be generated from mouse somatic cells through treatment with a combination of seven small-molecule compounds including DZNep, a histone methyltransferase inhibitor. ${ }^{15}$ Although inhibitors are useful for controlling epigenetic modifications, we believe that combining of gene transfection ${ }^{16,17}$ with inhibitor delivery could more efficiently control epigenetic modifications than treatment with inhibitors alone.
It could be accomplished by combining expression of epigenetic modification enzymes with delivery of inhibitors of enzymes that are involved in the reverse reactions (Figure 1a).

We designed an epigenetics control (EpC) carrier as a novel method to control histone acetylation through the synergistic effect of gene transfection and inhibitor delivery (Figure 1b). We expect that this method, if established, might make the control of cell differentiation and treatment of diseases possible by returning a system to its original epigenomic state. The resulting epigenetics engineering could potentially also be used as a treatment for intractable diseases.

To establish an EpC carrier for the control of histone acetylation, we focused on combining transfection of a histone acetyltransferase gene, in this case, the histone acetyltransferase CAF with delivery of an HDAC inhibitor, in this case, trichostatin A (TSA). To encapsulate both TSA and plasmid DNA encoding the CAF gene, polymer carriers were of interest because they could potentially contain both these components. In this report, we describe the development of a biodegradable EpC carrier to control histone acetylation. The developed EpC carrier comprises the following four components, (i) plasmid DNA encoding the CAF gene, (ii) the HDAC inhibitor TSA, (iii) the cationic lipid $N$-[1-(2,3-dioleoyloxy)propyl]$\mathrm{N}, \mathrm{N}, \mathrm{N}$-trimethylammonium methyl-sulfate $(\text { DOTAP })^{18}$ to complex with the plasmid DNA, and 4) poly (lactic acid) as a biodegradable polymer to encapsulate the components mentioned above. The resulting EpC carrier was expected to be able to control histone modification through the synergetic effect between gene transfection and delivery of the enzyme inhibitor. Additionally, the EpC carrier was expected to exhibit controlled release due to its biodegradability. Here, we report the effective control of histone acetylation through simultaneous transfection of the CAF gene with delivery of an HDAC inhibitor by using an EpC carrier.

\section{EXPERIMENTAL PROCEDURES}

Materials used to synthesize the EpC carrier Poly (lactic acid) with an average molecular weight of 10000 was used as a biodegradable polymer (PLA-0010, Wako, Japan). DOTAP was used as a cationic lipid and was purchased from Roche diagnostics (Tokyo, Japan). Plasmid DNA encoding the CAF gene (CMVpromoter) was purchased from the Riken Gene Bank (Ibaraki, Japan). TSA was purchased from Tokyo Chemical Industry (Tokyo, Japan).

\section{Cell culture}

Human leukemia HL60 cells were obtained from the Riken Cell Bank (Ibaraki, Japan). HL60 cells were maintained in RPMI 1640 containing 10\% heat-inactivated fetal bovine serum with antibiotics $\left(37^{\circ} \mathrm{C}, 5 \% \mathrm{CO}_{2}\right)$.

\section{Preparation of the EpC carrier}

First, plasmid DNA $(0.3 \mathrm{mg})$ was mixed with DOTAP $(0.7 \mathrm{mg})$ for an hour in phosphatebuffered saline $(500 \mu \mathrm{l})$. Then, PLA $(100 \mathrm{mg})$, TSA ( $1 \mathrm{mg})$ and the DNA/DOTAP complex were completely dissolved in a mixture of acetone $(2 \mathrm{ml})$ and methanol $(1 \mathrm{ml})$. The resulting organic solution was poured into $30 \mathrm{ml}$ of an aqueous poly(vinyl alcohol; $1.0 \% \mathrm{w} / \mathrm{v})$ and stirred at 400 r.p.m. for $5 \mathrm{~min}$. The entire dispersed system was centrifuged $(10000 \mathrm{~g}$ for $20 \mathrm{~min}$ ) and resuspended in distilled water. This process was repeated in triplicate. The resulting dispersion was freeze-dried. 

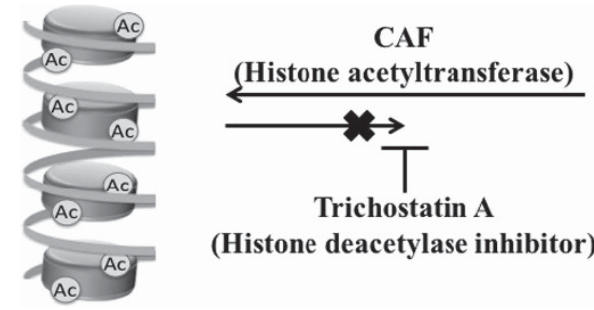

(Histone deacetylase inhibitor)

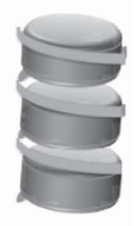

b

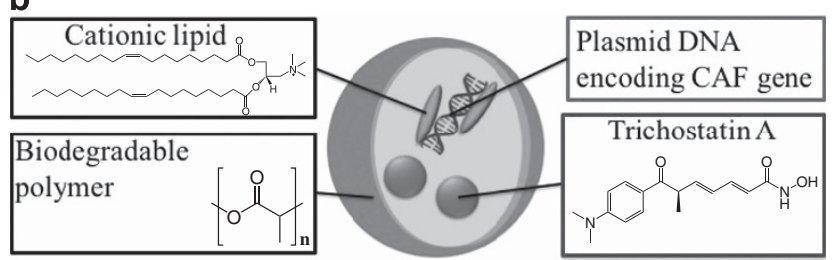

Epigenetics control carrier (EpC carrier)

Figure 1 Fundamental concept of the epigenetics control (EpC) carrier. (a) The concept of control of histone acetylation with the EpC carrier. (b) The EpC carrier comprises the following four components: (i) plasmid DNA encoding a CAF gene as a histone acetyltransferase, (ii) trichostatin A as a histone deacetylase inhibitor, (iii) a cationic lipid and (iv) poly(lactic acid). A full color version of this figure is available at Polymer Journal online.

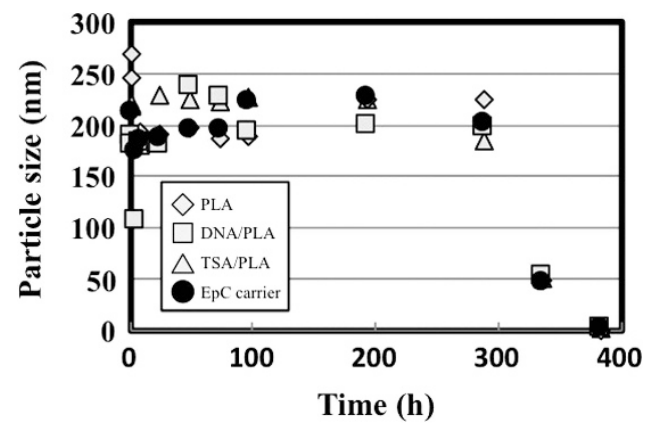

Figure 2 Time-dependent changes in the particle size of the EpC carrier as determined by DLS.

Table 1 Particle size and zeta potential of the EpC carrier and other samples

\begin{tabular}{lcc}
\hline Samples & $\begin{array}{c}\text { Particle size } \\
(\mathrm{nm})\end{array}$ & $\begin{array}{c}\text { Zeta potential } \\
(\mathrm{mV})\end{array}$ \\
\hline DNA/DOTAP & $101 \pm 25$ & +19 \\
DOTAP/PLA & $176 \pm 34$ & -11 \\
TSA/PLA & $191 \pm 23$ & -34 \\
DNA/DOTAP/PLA & $233 \pm 41$ & -35 \\
EpC carrier & $261 \pm 75$ & -28 \\
\hline
\end{tabular}

Abbreviations: DOTAP (cationic lipid), N-[1-(2,3-Dioleoyloxy) propyl]-N, N, N-trimethylammonium methyl-sulfate; EpC, epigenetics control carrier; PLA, poly(lactic acid); TSA, trichostatin A.

\section{Particle size and zeta potential of the EpC} carrier

The average particle size and zeta potential of the $\mathrm{EpC}$ carrier and other samples dispersed in phosphate-buffered saline were determined by dynamic light scattering (ELSZ2, Otsuka, Japan). Time-dependent changes of the particle sizes were measured from 0 to $384 \mathrm{~h}$.
Confirmation of drug encapsulation in the EpC carrier

The functional groups of TSA were analyzed by Fourier transform infrared spectroscopy with an FT-IR-6100 (JASCO, Tokyo, Japan). The functional groups of TSA, PLA, the mixture of TSA and PLA and the EpC carrier were determined by an attenuated total reflection method using Ze-Se crystals (ATR-PRO450S).

Loading efficiency of drug encapsulation in the EpC carrier

The prepared EpC carrier was dissolved in DMSO. The absorbance of TSA was measured by UV/Vis spectrometry. Finally, the amount of encapsulated TSA and the loading efficiency of TSA were calculated from the calibration curve.

\section{Evaluation of several proteins by western blotting}

The EpC carrier (final concentration of DNA and TSA were $200 \mathrm{ng}$ per well and $1 \mathrm{~nm}$, respectively) was added into HL60 cells $\left(1 \times 10^{5}\right.$ cells per well $)$ and incubated in RPMI 1640 containing 10\% heat-inactivated fetal bovine serum with antibiotics $\left(37^{\circ} \mathrm{C}, 5 \% \mathrm{CO}_{2}\right)$ for 2 to 16 days. The cells were lysed, and a reducing agent was added to break the disulfide bonds. The resulting samples were separated by SDS-polyacrylamide gel electrophoresis. The resulting gel was electro-transferred to a polyvinylidene difluoride membrane. Then, the membrane was blocked using $5 \%$ fat-free milk. After blocking, the sample was incubated with primary antibody and secondary antibodies. Finally, the target proteins were detected by an ECL prime western blotting detection system (GE Healthcare). The following primary antibodies specific to the following proteins were used for western blotting analysis: CAF antibody (Cell Signaling Technology, Tokyo, Japan), acetylated histone H3-K9, K18, K27 antibody (Cell Signaling Technology) and a betaActin antibody (Cell Signaling Technology). An horseradish peroxidase-linked antibody (Cell Signaling Technology) was used as a secondary antibody. The chemiluminescence of the bands was detected with an image analyzer (AE-9300 EzCapture MG, ATTO, Tokyo, Japan).

\section{RESULTS AND DISCUSSION}

First, we evaluated the particle size and zeta potential of the EpC carrier by dynamic light scattering (Table 1). The particle size and zeta potential of DNA/DOTAP complex were determined to be $101 \pm 25 \mathrm{~nm}$ and $+19 \mathrm{mV}$, respectively. In the case of TSA/PLA and DNA/PLA, the particle sizes were $191 \pm 23 \mathrm{~nm}$ and $33 \pm 41 \mathrm{~nm}$, respectively, and the zeta potentials were $-34 \mathrm{mV}$ and $-35 \mathrm{mV}$, respectively. For DOTAP/PLA, the particle size and zeta potential were $176 \pm 34 \mathrm{~nm}$ and $-11 \mathrm{mV}$, respectively. The corresponding values for the EpC carrier were $261 \pm 75 \mathrm{~nm}$ and $-28 \mathrm{mV}$, respectively. Because the EpC carrier demonstrated an increase in particle size and had a negative charge, the DNA/DOTAP complex was considered to be encapsulated in the PLA nanoparticles.

Time-dependent changes in the particle size of the EpC carrier were determined by dynamic light scattering (Figure 2). The particle size of the EpC carrier was maintained for 12 days $(288 \mathrm{~h})$. After incubation for $288 \mathrm{~h}$, the particle size of the EpC carrier decreased below $50 \mathrm{~nm}$, suggesting that the nanoparticles were biodegradable. Therefore, controlled release of the drug was considered to occur after $288 \mathrm{~h}$ of incubation.

Drug encapsulation in the EpC carrier was confirmed by FT-IR spectroscopy because surface information could be obtained from the FT-IR spectra (Figure 3). Attribution of the FT-IR spectrum of TSA was carried out as described in previous reports. ${ }^{19}$ In TSA, monocyclic aromatic C-H out-of-plane deformation vibrations at $1525-1475 \mathrm{~cm}^{-1}$ were observed, and the peaks at $1390-1370 \mathrm{~cm}^{-1}$ were attributed to aromatic $\mathrm{C}-\mathrm{C}$ stretching 
a

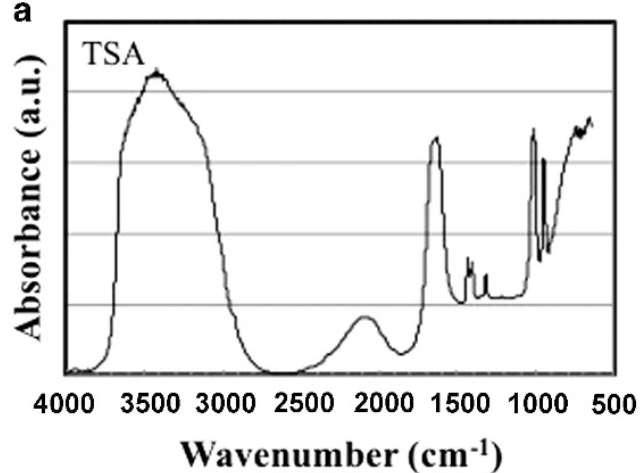

c

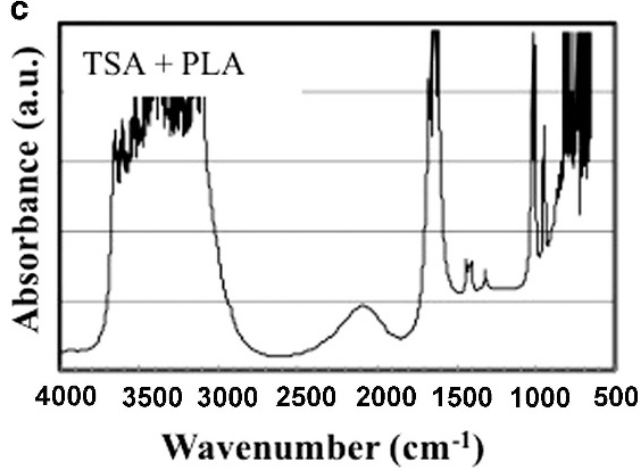

b

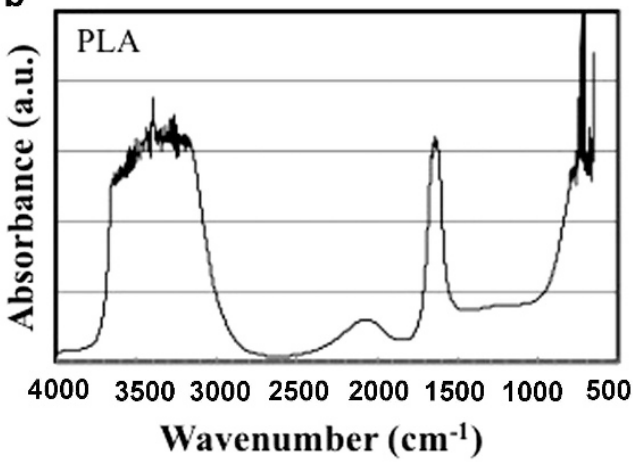

d

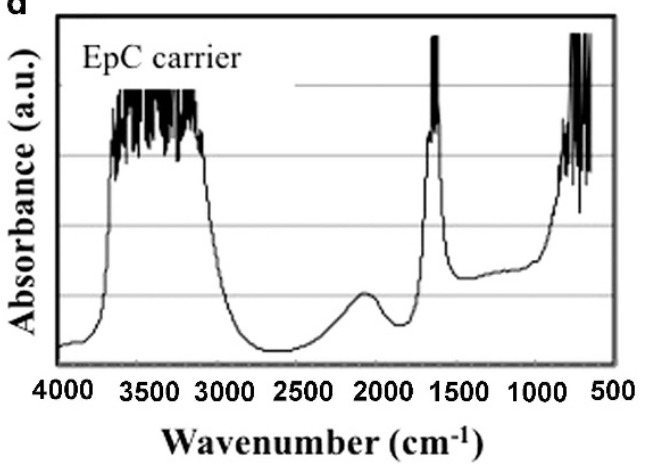

Figure 3 (a, b) FT-IR spectra of TSA and poly(lactic acid) (PLA). (c) Spectrum of the TSA and PLA mixture. (d) Spectrum of the EpC carrier.

a

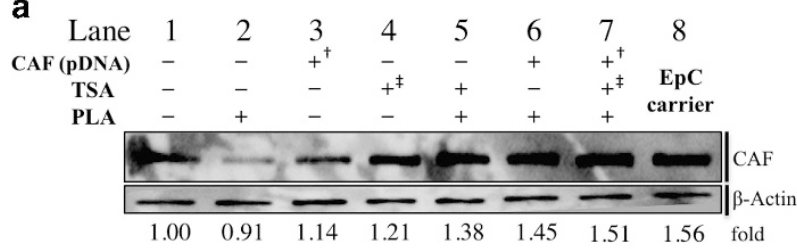

b
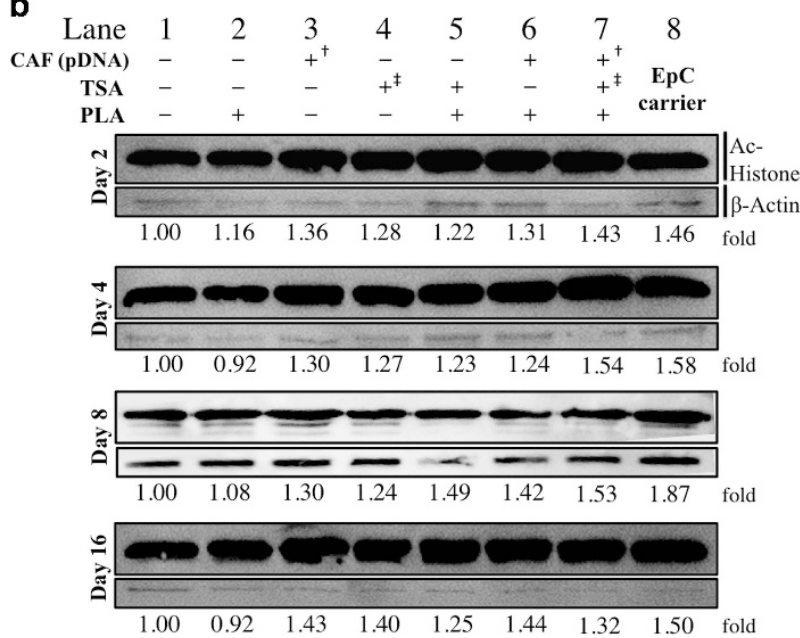

Figure 4 (a) The protein level of CAF was evaluated by western blotting. (b) Amount of histone acetylation of the cells treated with the EpC carrier containing TSA and plasmid DNA encoding the CAF gene at 2, 4, 8 and 16 days. ${ }^{\dagger} \mathrm{CAF}$ (pDNA) was transfected with Lipofectin. ${ }^{\ddagger}$ TSA was dissolved in DMSO and added to the cells. vibrations. Aromatic tertiary amine $\mathrm{C}-\mathrm{N}$ stretching vibrations were also observed at $1340-1250 \mathrm{~cm}^{-1}$. Alkene out-of-plane deformation vibrations were observed at $980-900 \mathrm{~cm}^{-1}$ (Figure 3a). Similarly, in the mixture of TSA and PLA nanoparticles, these peaks were also detected because TSA was not encapsulated in the PLA nanoparticles (Figure 3c). However, these peaks were not observed in the PLA nanoparticles because TSA was not present (Figure 3b). No peaks were observed in the region of $1000-1500 \mathrm{~cm}^{-1}$, as shown in Figure 3d, and TSA was considered to be incorporated into the EpC carrier. Furthermore, the specific UV/Vis spectrum of TSA, detected for samples with the EpC carrier dissolved in DMSO, supports the incorporation of TSA (Supplementary Figure S1). Collectively, these results suggest that TSA was encapsulated into the carrier.

Next, we evaluated the loading efficiency of TSA in the EpC carrier by UV/Vis spectrometry. We prepared the EpC carrier in a feed concentration of $495 \mathrm{~nm}$ TSA per $15 \mathrm{mg}$ of the EpC carrier. The absorbance $(340 \mathrm{~nm})$ of TSA in the EpC carrier was 0.454 (Supplementary Figure S1B). The TSA calibration curve (Supplementary Figure S1A) 
indicated that the loading concentration of TSA in the EpC carrier was 378 nm. From these results, the loading efficiency of the EpC carriers was estimated to be $76.4 \%$.

To confirm whether the transfected CAF gene was expressed in HL60 cells, we performed western blotting after incubation for 2 days (Figure 4a). The cells treated with the EpC carrier demonstrated the highest expression of CAF (Figure 4a lane 8). In particular, use of the EpC carrier resulted in higher CAF expression than sid the separate transfection of the CAF gene and delivery of TSA (Figure 4a lane 7). The amounts of the CAF gene and TSA in the same cell treated with EpC carrier exceeded that in the sample with separate transfection of the CAF gene and delivery of TSA. The increased amount of both the CAF gene and TSA in the same cell may have led to an increase in endogenous CAF protein. Taking these results into account, we concluded that the EpC carrier contained both pDNA and TSA.

Next, we examined whether the resulting EpC carrier controlled histone acetylation. Figure $4 \mathrm{~b}$ shows the amount of histone acetylation in the HL60 cells at each day of the experiment. The amount of acetylated histone was normalized to the $\beta$-actin band. The amounts in all the samples were compared with the amount in the control (Figure $4 \mathrm{~b}$ lane 1). There was not a significant increase in the level of histone acetylation of the cells treated with PLA (Figure 4b lane 2) after 2 days. In the case of transfect CAF gene transfection with Lipofectin, the amount of acetylated histone was increased by 1.36 -fold (Figure 4b lane 3). The cells treated with TSA demonstrated an increase in histone acetylation of 1.28-fold (Figure $4 \mathrm{~b}$ lane 4 ), and the cells treated with the PLA nanoparticles containing TSA also exhibited an increase of 1.22-fold (Figure 4b lane 5). Similarly, the PLA nanoparticles containing the CAF/DOTAP complex led to an increase of 1.31-fold (Figure $4 \mathrm{~b}$ lane 6). When we separately transfected the CAF gene with Lipofectin and delivered TSA dissolved in DMSO and PLA nanoparticles, the amount of acetylated histone increased by 1.43 -fold (Figure $4 \mathrm{~b}$ lane 7 ). It should be noted that HL60 cells treated with the EpC carrier demonstrated the highest increase and exhibited a 1.46-fold in histone acetylation levels (Figure 4b lane 8). CAF is known to control histone acetylation competitively with HDAC3, as previously described. ${ }^{20}$ TSA affects all HDACs. An increased amount of both CAF and TSA in the same cell would lead to an increase in histone acetylation. Therefore, the EpC carrier succeeded in facilitating histone acetylation, whereas inhibiting histone deacetylation. Notably, the amount of histone acetylation of the cells treated with the EpC carrier was maintained after 16 days (1.50-fold increase) compared with that in cells subjected separately to CAF gene transfection and TSA delivery without the EpC carrier (1.32-fold increase). Epigenetic states are known to be inherited through cell division. The simultaneous control of histone acetylation by CAF and TSA suggests that the EpC carrier may have affected cell differentiation and resulted in retention of a large amount of acetylated histone. These results suggest that the biodegradability of the EpC carrier mentioned in Figure 2 affects the maintenance of histone acetylation.

In conclusion, we demonstrated the development of a new biodegradable nanoparticle, which we have named the EpC carrier. Continuous induction of histone acetylation was achieved by simultaneous gene transfection and inhibitor delivery by using the EpC carrier. This is the first report on the utilization of an EpC carrier as a unique tool in the epigenetics field.

\section{CONFLICT OF INTEREST}

The authors declare no conflict of interest.

Yutaro Asaba, Shoichiro Asayama and Hiroyoshi Kawakami

Department of Applied Chemistry, Tokyo Metropolitan University, Tokyo, Japan E-mail: asayama-shoichiro@tmu.ac.jp or kawakami-hiroyoshi@tmu.ac.jp

1 Weinhold, B. Epigenetics: the science of change. Environ. Health Perspect. 114, A160-A167 (2006).

2 Biel, M., Wascholowski, V. \& Giannis, A. Epigenetics - an epicenter of gene regulation: histones and histone-modifying enzymes. Angew. Chem. 44, 3186-3216 (2005).
3 Miller, C. A. \& Sweatt, J. D. Covalent modification of DNA regulates memory formation, neuron. Neuron $\mathbf{5 3}$, 857-869 (2007).

4 Zentner, G. E. \& Henikoff, S. Regulation of nucleosome dynamics by histone modifications. Nat. Struct. Mol. Biol. 20, 259-266 (2013).

5 Nakamura, T., Liu, Y. J., Nakashima, H., Umehara, H., Inoue, K., Matoba, S., Tachibana, M., Ogura, A., Shinkai, Y. \& Nakano, T. Regulation of nucleosome dynamics by histone modifications. Nat. Struct. Mol. Biol. 486, 415-419 (2012).

6 Rando, T. A. \& Chang, H. Y. Aging, rejuvenation, and epigenetic reprogramming: resetting the aging clock. Cell 148, 46-57 (2012).

7 Dolinoy, D. C., Huang, D. \& Jirtle, R. L. Maternal nutrient supplementation counteracts bisphenol A-induced DNA hypomethylation in early development. PNAS 104, 13056-13061 (2007).

8 Sandovici, I., Hammerle, C. M., Ozanne, S. E. \& Constância, M. Developmental and environmental epigenetic programming of the en-docrine pancreas: consequences for type 2 diabetes. Cell. Mol. Life Sci. 70, 1575-1595 (2013).

9 Dawson, M. A. \& Kouzarides, T. Cancer epigenetics: from mechanism to therapy. Cell 150, 12-27 (2012).

10 New, M., Olzscha, H. \& La Thangue, N. B. HDAC inhibitor-based therapies: can we interpret the code? Mol. Oncol. 6, 637-656 (2012).

11 Balch, C., Yan, P., Craft, T., Young, S., Skalnik, D. G., Huang, T. H. \& Nephew, K. P. Antimitogenic and chemosensitizing effects of the methylation inhibitor zebularine in ovarian cancer. Mol. Cancer Therap. 10, 1505-1514 (2005).

12 Marks, P. A. \& Breslow, R. Dimethyl sulfoxide to vorinostat: development of this histone deacetylase inhibitor as an anticancer drug. Nat. Biotechnol. 25, 84-90 (2007).

13 Winkley, M. W. \& Robins, R. K. Direct glycosylation of 1,3,5-triazinones. A new approach to the synthesis of the nucleoside antibiotic 5-azacytidine (4-amino-1beta-D-ribofuranosyl-1,3,5-triazin-2-one) and related derivatives. J. Org. Chem. 35, 491-495 (1970).

14 Huangfu, D., Maehr, R., Guo, W., Eijkelenboom, A., Snitow, M., Chen, A. E. \& Melton, D. A. Induction of pluripotent stem cells by defined factors is greatly improved by small-molecule compounds. Nat. Biotechnol. 26, 795-797 (2008).

15 Hou, P., Li, Y., Zhang, X., Liu, C., Guan, J., Li, H., Zhao, T., Ye, J., Yang, W., Liu, K., Ge, J., Xu, J., Zhang, Q., Zhao, Y. \& Deng, H. Pluripotent stem cells induced from mouse somatic cells by small-molecule compounds. Science 341, 651-654 (2013).

16 Asayama, S., Nishinohara, S. \& Kawakami, H. Zinc-chelated poly(1-vinylimidazole) and a carbohydrate ligand polycation form DNA ternary complexes for gene delivery. Bioconjug. Chem. 22, 1864-1868 (2011).

17 Asayama, S., Hakamatani, T. \& Kawakami, H. Synthesis and characterization of alkylated poly(1-vinylimidazole) to control the stability of its DNA polyion complexes for gene delivery. Bioconjug. Chem. 21, 646-652 (2010).

18 Simões, S., Slepushkin, V., Gaspar, R., Lima, M. C. \& Düzgüneş, N. Gene delivery by negatively charged ternary complexes of DNA, cationic liposomes and transferrin or fusigenic peptides. Gene Ther. 5, 955-964 (1998).

19 Tsuji, N., Kobayashi, M., Nagashima, K., Wakisaka, Y. \& Koizumi, K. A new antifungal antibiotic, trichostatin. J. Antibiot. 29, 1-6 (1976).

20 García, N. B., Juan, E. A., Cruz, X. \& Balbás, M. Autoacetylation regulates P/CAF nuclear localization. J. Biol. Chem. 284, 1343-1352 (2009).

Supplementary Information accompanies the paper on Polymer Journal website (http://www.nature.com/pj) 